\title{
Entre la evolución, la psicología y la política: Luis López de Mesa, el primer psicólogo colombiano
}

\author{
Rubén Ardila \\ Universidad Nacional de Colombia
}

Recibido: 15 de abril de 2014 / Aprobado: 30 de abril de 2014

Luis López de Mesa (1884-1967) es uno de los principales pensadores colombianos. Estudió Medicina en la Universidad Nacional de Colombia y se graduó en 1912. Cursó estudios de especialización en la Universidad de Harvard en Psicología y Psiquiatría; posteriormente, viajó por varios países de Europa en los que visitó diversos centros de investigación. En Colombia se desempeñó como psiquiatra, profesor universitario, filósofo y escritor. Ocupó importantes cargos en la política, como el de ministro de Educación, ministro de Relaciones Exteriores, senador de la República y rector de la Universidad Nacional de Colombia, entre otros cargos. Investigó sobre evolución y buscó difundir las ideas de Darwin y Spencer. Entre sus principales libros que abordan temas psicológicos se encuentran Nosotros y la Esfinge (1947), De cómo se ha formado la nación colombiana (1934) y varias novelas psicológicas.

\section{López de Mesa / evolución / Colombia / cultura latinoamericana}

\section{Between evolution, psychology and politics: Luis López de Mesa, the first} Colombian psychologist

Luis López de Mesa (1884-1967) is considered as one of the main Colombian thinkers. He studied Medicine at the National University of Colombia and got his degree in 1912. He did graduate work at Harvard University in psychology and psychiatry. He traveled for several European countries visiting research centers Upon his return to Colombia he worked as a psychiatrist, University professor, philosopher and writer. He occupied important political positions, including Minister of Education, Minister of Foreign Affairs, Senator of the Republic, Rector of the National University of Colombia, and others. López de Mesa did research on evolution and introduced Darwin's and Spencer's concepts and ideas in scientific circles. Among his main books that deal with psychological topics there are Nosotros y la Esfinge [We and the Sphinx] (1947), De cómo se ha formado la nación colombiana [How the Colombian Nation was formed] (1934), and several psychological novels.

López de Mesa / evolution / Colombia / Latin American culture 


\section{SU VIDA Y SU OBRA}

López de Mesa es una de las figuras más originales y creativas de la cultura colombiana. Realizó aportes de importancia a la psicología, la filosofía, la sociología, la historia de la cultura, la literatura y la organización social. Nació en un pueblo de Antioquia llamado Donmatías (que en ese entonces se denominaba Azuero), estudió Medicina en la Universidad Nacional de Colombia en Bogotá y se graduó en 1912. Trabajó como profesor de Historia de la Medicina, de Sociología Americana y de Historia del Arte entre 1912 y 1916, antes de viajar a los Estados Unidos para cursar estudios de posgrado. Se especializó en Psicología y Psiquiatría en la Universidad de Harvard, en la época en que la psicología, en esa universidad, se centraba en las ideas de Münsterberg, McDougall y otras figuras de relevancia mundial en la historia de esta disciplina.

Entre 1918 y 1922 completó sus estudios en Inglaterra y Francia, y luego viajó por Europa. Regresó a Colombia y estableció su consultorio como psiquiatra; allí se dedicó a escribir libros y a organizar sus conceptualizaciones acerca de la cultura humana, la sociedad, el psiquismo, la raza, la historia, la evolución y otros temas de amplio espectro. $\mathrm{Su}$ primer libro publicado se titula $E l$ libro de los apólogos (1918). Otras de sus obras que merecen destacarse son Civilización contemporánea (1926), De cómo se ha formado la nación colombiana (1934), Disertación sociológica (1939), Nosotros y la Esfinge (1947), Perspectivas culturales (1949), Escrutinio sociológico de la historia colombiana (1956) y Oraciones panegíricas (1964). Publicó también dos novelas psicológicas que demuestran la profundidad de su pensamiento como psicólogo: La tragedia de Nilse (1928) y Biografía de Gloria Etzel (1929).

Su trabajo en psicología se encuentra en varias de sus obras. En una de ellas (1956) se refiere a los siguientes aspectos de esta disciplina:

[La] psicología humana normal y patológica, el comportamiento instintivo de los animales y su probable curso filogenético, habida cuenta de las formas, cantidades y estructuras que lo condicionan [...] la base emotiva de la cultura iberoamericana, la diversa y compleja psicología de los mulatos y mestizos, la unidad de génesis del instinto y la inteligencia, el concepto de conciencia intelectiva [...] (p. 304).

De López de Mesa se afirma "incluso que fue el precursor entre nosotros de los tests psicológicos, que serían implantados dos años más tarde por doña Mercedes Rodrigo y por el doctor Alfonso Esguerra Gómez" (Medina Muñoz, 2000, p. 237).

En una mesa redonda sobre la raza colombiana, que tuvo lugar en 1920 , López de Mesa se refiere a la persona- 
lidad e inteligencia de los colombianos y afirma:

La parte intelectual me merece un mejor concepto. Somos un pueblo bien dotado en este sentido y aun pudiera decirse muy bien dotado [...]. Mis materias de experimentación fueron el texto y las pruebas de Yerkes-Bridges-Hardwick, el texto y las pruebas de Terman, y unos cuadros de mi propia iniciativa para obtener una adaptación más rigurosa al medio psíquico colombiano.

El test al que se alude, el primero producido por un autor colombiano, lamentablemente se ha perdido. En varias ocasiones, López de Mesa se definió a sí mismo como "psicólogo", y podemos decir que fue la primera persona que se identificó como tal en Colombia.

Otras actividades en estos campos fueron su trabajo como profesor de Psiquiatría en la universidad, la dirección general -ad honórem - de los manicomios de Bogotá y su participación en muchos eventos de psicología. López de Mesa era rector de la Universidad Nacional de Colombia cuando se inauguró formalmente la enseñanza profesional de la psicología en el país, el 9 de julio de 1948 (la carrera se había creado como parte del Instituto de Psicología Aplicada el 20 de noviembre de 1947; los primeros estudiantes comenzaron clases en febrero de 1949).

López de Mesa participó activamente en el mundo científico colombia- no e internacional, como en la Academia Colombiana de Ciencias Exactas, Físicas y Naturales (donde fue elegido miembro numerario en 1937); en la Academia Colombiana de la Lengua; en la Academia Nacional de Medicina; en el Colegio Máximo de Academias, etc. Trabajó sobre evolución biológica y analizó en detalle la obra de Darwin y de Spencer, entre otros. Ocupó numerosos cargos públicos, vinculados todos con la educación y con las relaciones internacionales. Creó la Biblioteca de Cultura Aldeana para difundir la cultura a lo largo y ancho del país. Fue ministro de Educación durante la administración de Alfonso López Pumarejo en 1934, y ministro de Relaciones Exteriores en 1938, durante la administración de Eduardo Santos. Participó en la Conferencia Internacional Americana, reunida en Lima, y en varias otras conferencias latinoamericanas e interamericanas, en las cuales siempre ocupó un papel protagónico y de liderazgo. Promovió la cultura popular, la ciencia, la tecnología, la navegación por los ríos del país y la industrialización. Volvió a ser ministro de Relaciones Exteriores en 1945, durante la administración de Alberto Lleras Camargo. Fue rector de la Universidad Nacional de Colombia en 1948, como señalamos anteriormente.

En medio de todas estas actividades públicas, tuvo tiempo para escribir libros de filosofía, sociología, historia de la humanidad y otros tópicos de gran envergadura. Afirmó en alguna oca- 
sión que tanto trabajo intelectual no le había dejado tiempo para casarse. Sus libros, sus artículos y su participación en eventos nacionales e internacionales estuvieron a la vanguardia de la ciencia y la cultura de su tiempo, y demostraron la enorme erudición de su autor. $\mathrm{Su}$ libro Nosotros y la Esfinge (1947) es, seguramente, una de las obras de filosofía más originales producidas por un autor colombiano.

La cultura colombiana, las perspectivas del país, sus determinantes psicológicos, la posibilidad de contribuir a la cultura universal desde este país, fueron temas de gran preocupación en su obra. Se le ha acusado de determinista, de panteísta y de ser un filósofo poco sistemático. Sin embargo, se ha afirmado que, a pesar de todas las limitaciones, su aporte central fue el siguiente:

[...] López de Mesa pareció echar sobre sus hombros la tarea de probar que nosotros teníamos la potencia y las virtualidades para construir una gran nación y por ello luchó, triunfó y sufrió en su vida [...]. Nos invitó a pensar por nuestra cuenta y a pensar en grande (Uribe Celis, 1985, p. 284).

Desde el punto de vista negativo, hay varios elementos que es necesario señalar. Sus ideas tuvieron poca influencia en la cultura popular colombiana, menos de la que López de Mesa hubiera deseado, pues se las consideró extremadamente sofisticadas, avanzadas y refinadas para la época. Uno de sus mejores interlocutores fue el filósofo Julio Enrique Blanco (1890-1986), con quien sostuvo una "correspondencia filosófica" entre 1917 y 1966, o sea, prácticamente durante toda la vida intelectual de López de Mesa. El libro que recoge dicha correspondencia (Blanco \& López de Mesa, 1987) es una valiosa fuente primaria sobre las ideas filosóficas y también psicológicas de estos dos pensadores colombianos.

Otro punto oscuro de la vida de López de Mesa fue su posición ante la inmigración de judíos al país. Siendo ministro de Relaciones Exteriores en la administración de Eduardo Santos, durante la Segunda Guerra Mundial, se opuso a la inmigración de judíos. Esto influyó negativamente en la posibilidad de dar asilo y oportunidades de vida a los perseguidos por los nazis. El presidente Eduardo Santos, por el contrario, estuvo a favor de la inmigración. Un considerable número de judíos pudo emigrar a Colombia, al igual que a otros países del continente americano. Las ideas de López de Mesa sobre la "raza" no estuvieron a la altura de los desarrollos posteriores de la ciencia, a diferencia de sus otras conceptualizaciones.

La obra de López de Mesa, a pesar de esas importantes limitaciones, puede considerarse un aporte original y que ha trascendido la época y los prejuicios de ese momento histórico. Veamos la contribución de su libro de 1934 a la psicología colombiana. 


\section{DE CÓMO SE HA FORMADO LA NACIÓN COLOMBIANA}

Según López de Mesa, para entender al país, su gente, su estado actual y sus perspectivas es preciso comenzar con sus ancestros como pueblo. Para ello analiza la influencia de los grupos culturales que forman la sociedad colombiana, incluyendo a los habitantes originarios, los conquistadores ibéricos, los africanos que llegaron al país como esclavos. Describe la influencia del clima, a la manera de Francisco José de Caldas. Analiza los factores positivos que han dejado los distintos grupos culturales y "raciales", así como la manera en que se han integrado en los distintos niveles climáticos de Colombia. Señala sus limitaciones, sus problemas, la influencia de la transmisión cultural. Se preocupa por nuestro futuro como sociedad y se pregunta al respecto: “Bastarían estas cualidades, la universalidad, la simpatía, la plasticidad, la generosidad y el sentido estético para crear un gran pueblo histórico, es decir, un pueblo que añada algo espiritual a la evolución del linaje humano?" (López de Mesa, 1970 [1934], p. 18).

Aunque su respuesta es positiva, se preocupa también por los problemas asociados con la pereza que observa en los descendientes de españoles, en la gente de origen africano y en ciertas ramas indígenas. Esto no depende únicamente de la cultura ni únicamente de la raza, dado que hay innumerables excepciones. Señalemos, por otra parte, que en la época en que se escribe este libro, entre las dos guerras mundiales, los problemas asociados con el concepto de "raza" tenían una gran importancia. Se pensaba que había razas superiores e inferiores, grupos étnicos que habían aportado a la civilización humana e iban a seguir haciéndolo, y otros que no lo habían hecho ni lo harían nunca. Ese "determinismo genético" de las décadas de los años 1920, 1930 y 1940 es una preocupación de López de Mesa, quien se refiere a los problemas derivados del concepto de "raza" en numerosas ocasiones. Sin embargo, la teoría de base es diferente, como se observa en su énfasis en la educación campesina, la nutrición, la solución de los problemas de salud pública (el alcoholismo, la prevención de las enfermedades), en el papel civilizador de las academias y las universidades, la cultura popular y otros.

La raza colombiana, mezcla de varias etnias, además de ser generosa, interesada en la estética y en la universalidad, es también una raza propensa a la depresión y la ciclotimia, melancólica y con inadecuada disciplina. En su libro pasa revista a las distintas regiones, sociedades, culturas, y se centra entre otras en la cultura de los Santanderes y en la cultura de Antioquia.

Otros capítulos sobre economía, constitucionalidad, religión y religiosidad, arte, empresas culturales, son igualmente importantes. En todos ellos se propone una mirada psicológica y 
respuestas a los problemas desde la perspectiva de la psicología. Sin duda, este libro es uno de los primeros esfuerzos por hacer una psicología social en nuestro país.

En el contexto en que se escribió este libro, la teoría de la evolución, las nuevas tendencias artísticas, el psicoanálisis para el caso de la psicología, las diferencias entre las personas y entre los grupos, la crisis económica que acababa de terminarse, los avatares de Europa entre las dos guerras mundiales, el surgimiento de Estados Unidos como potencia emergente, en muchos aspectos como balance a la hegemonía europea, todo esto tenía gran relevancia. Era una época de grandes transformaciones, de crisis, de tratar de recuperarse de la Primera Guerra Mundial y de mirar con desconfianza e incertidumbre el futuro cercano.

El libro busca analizar problemas locales de Colombia, con la mirada puesta en el mundo como un todo que se estaba transformando a pasos agigantados y que estaba lejos de encontrar alguna ruta que pudiera considerarse definitiva. Debe entenderse en el contexto de su época y de las circunstancias que rodearon su génesis. No puede considerarse una obra de psicología científica, en sentido estricto, con la metodología y las teorías que utiliza esta disciplina en nuestros días, sino que debe valorar- se como obra pionera, altamente creativa, obra de uno de los pensadores que más se interesó por su país, por su época y por la perspectiva que la psicología brindaba para el estudio de los grandes problemas del mundo.

\section{RefERENCIAS}

Blanco, J. E., \& López de Mesa, L. (1987). Correspondencia filosófica (1917-1966). Barranquilla: Ediciones Uninorte.

López de Mesa, L. (1956). Escrutinio sociológico de la historia colombiana. Bogotá: Academia Colombiana de Historia.

López de Mesa, L. (1970) [1934]. De cómo se ha formado la nación colombiana. Medellín: Editorial Bedout.

Medina Muñoz, L. R. (2000). Tradición académica. Diccionario biográfico y bibliográfico de la Academia Colombiana de Ciencias Exactas, Físicas y Naturales. Bogotá: ACCEFYN.

Uribe Celis, C. H. (1985). Luis López de Mesa, aproximación crítica a su obra. En F. M. Velásquez, C. H. Uribe Celis, \& E. Santa. Vida y obra del profesor Luis López de Mesa (pp. 173-293). Medellín: Editorial Universidad de Antioquia. 